\title{
Design and optimization on rootstock cutting mechanism of grafting robot for cucurbit
}

\author{
Kai Jiang ${ }^{1}$, Qian Zhang ${ }^{2}$, Liping Chen ${ }^{1 *}$, Wenzhong Guo ${ }^{1}$, Wengang Zheng ${ }^{1}$ \\ (1. Beijing Research Center of Intelligent Equipment for Agriculture, Beijing 100097, China; \\ 2. Beijing Research Center of Information Technology for Agriculture, Beijing 100097, China)
}

\begin{abstract}
Cutting mechanisms in existing grafting machines are unable to completely cut through the rootstock growth point and can easily damage seedlings. During the mechanical operation of splice grafting, the cutting angle of the rootstock is an essential factor for ensuring the quality and survival rate of grafting seedlings and a stable process for grafting robots. Therefore, in this study, commonly used grafting rootstocks, e.g., cucurbita moschata, and calabash gourd were used as research objects for studying and analyzing the cutting angle of a splice grafting method. The morphological and structural parameters of the rootstock and scion were measured, and a structural model of the internal cavity of the rootstock was constructed using an image analysis method. The critical cutting angles for the cucurbita moschata and calabash gourd seedlings were obtained. According to the analysis, the grafting cutting angles for cucumber seedlings matching with cucurbita moschata seedlings were $20^{\circ}$ and $25^{\circ}$, respectively, and the fitting rate of the cutting surface of the rootstock and scion was $99.04 \%$. A cutting mechanism for the rootstock growth point and geometric model of the cutting operation were established, and the structural parameters of the mechanism and cutting angle adjustment were optimized. A cutting performance test showed that the success rate of the pressing the cotyledons of cucurbita moschata seedlings was $96.67 \%$, and the success rate of cutting was 98\%. The cutting accuracy was $96.8 \%$, and the cutting surface fitting rate of the rootstock and scion was $98.61 \%$. The latter differed by $0.43 \%$ from the theoretical rate but met the requirements for the splice grafting method. Thus, this study can provide a reference for the design of a cutting mechanism for a grafting robot.
\end{abstract}

Keywords: grafting robot, rootstock cavity, cutting angle, matched grafting, parameter optimization, cucurbit DOI: $10.25165 /$ j.ijabe.20201305.5803

Citation: Jiang K, Zhang Q, Chen L P, Guo W Z, Zheng W G. Design and optimization on rootstock cutting mechanism of grafting robot for cucurbit. Int J Agric \& Biol Eng, 2020; 13(5): 117-124.

\section{Introduction}

Grafting is an important part of the production process of industrial seedlings, and the corresponding labor accounts for $20 \%-30 \%$ of the entire process of seedling cultivation ${ }^{[1]}$. Therefore, seedling enterprises invest a significant amount of money to hire professional grafting workers every year. However, owing to long-term work under high-temperature and high-humidity conditions, grafting workers are prone to rheumatism and other diseases, making workers reluctant to engage in grafting work. This situation has led to a growing shortage of labor force in the seedling industry ${ }^{[2-5]}$. Grafting equipment can overcome the shortcomings (e.g., low efficiency and nonstandard grafting quality) of artificial grafting, and even ordinary workers without special professional training can operate the machinery, providing important technical support for machine grafting instead of manual grafting ${ }^{[6,7]}$. Therefore, in recent years, technology research on grafting robots has attracted significant attention from scholars at home and abroad ${ }^{[8-12]}$ and has become a research hotspot

Received date: 2020-03-29 Accepted date: 2020-07-31

Biographies: Kai Jiang, $\mathrm{PhD}$, Senior Engineer, research interest: agricultural intelligent equipment and agricultural robot, Email: jiangk@nercita.org.cn; Qian Zhang, Master, Research Assistant, research interest: agricultural informatization, Email: zhangq@nercita.org.cn; Wenzhong Guo, PhD, Researcher, research interest: facility gardening equipment, Email: guowz@ nercita.org.cn; Wengang Zheng, PhD, Researcher, research interest: agricultural informatization and automatic control, Email: zhengwg@nercita.org.cn.

*Corresponding author: Liping Chen, PhD, Researcher, research interest: precision agriculture. Beijing Research Center of Intelligent Equipment for Agriculture, Beijing 100097, China. Tel: +86-10-51503425, Email: chenlp@nercita.org.cn. in the field of agricultural robots ${ }^{[13-15]}$.

In China, splice grafting and plug-in grafting are two commonly used methods for melon grafting ${ }^{[16]}$. Among them, the operation for plug-in grafting is relatively simple (e.g., without a fixed clamp), but requires professional workers with skilled techniques. In addition, when using plug-in grafting, the grafting seedling age needs to be strictly controlled, to ensure the size requirements for rootstock and scion transplanting. After healing, the rootstock growth point must have the sprouting removed, increasing the workload. As splice grafting requires the rootstock growth point and cotyledon to be removed at once, after the operation using this method, the standard flatness of the incision angle is good, the docking fit is high, and the grafted seedlings can be synthesized quickly. Moreover, there is no removal of germination in the later stages of healing management. Based on a comprehensive consideration of grafting quality and efficiency, it has been concluded that it is difficult to realize plug-in grafting by machines, as it is highly dependent on artificial grafting, and cannot adapt to the long-term development of factory grafting and seedling raising enterprises. In contrast, the splice grafting method has advantages on mechanical standardization, has lower requirements for seedling age, and is more suitable for factory grafting and seedling raising ${ }^{[17]}$.

As there is a cavity in the hypocotyl of a rootstock section, it is necessary to ensure that the cavity is not cut during the grafting cutting process. If the cavity exposed from the rootstock section fits the scion section, the new roots will penetrate the cavity and enter the soil during the healing period of the scion seedlings, leading to failures in the disease resistance of the grafted seedlings ${ }^{[18,19]}$. As a result, to ensure the success of the grafting 
process, the rootstock must avoid the standardized incision formed by the cavity. When an appropriate cutting angle is determined for the rootstock in the splice method, it can avoid the exposure of the rootstock incision from the cavity and realize matched grafting of the rootstock and scion. This provides an important guarantee, i.e., for the mechanical grafting to improve the grafting accuracy and quality ${ }^{[20-22]}$.

This study analyzed the rootstock cutting angle in the process of splice grafting. First, by using image analysis technology, the cavity structure of a melon rootstock was measured and studied, and a critical cutting angle of the rootstock and a cutting angle of the matched grafting of the rootstock and scion were determined. Using cucurbita moschata and cucumber seedlings as test objects, a rootstock growth point cutting mechanism was developed, and its structural parameters were optimized. A cutting performance test was conducted to verify the rationality of the design. The research results provide a reference for the cutting mechanism design of a grafting robot.

\section{Cutting mechanism of rootstock}

\subsection{Cavity structure in rootstock}

In this study, Jingxin No. 8 rootstock cucurbita moschata and Jingxin No. 1 rootstock calabash gourd were selected as the test objects. The seeds were immersed in warm water at $50^{\circ} \mathrm{C}-55^{\circ} \mathrm{C}$ for $12 \mathrm{~h}$; subsequently, the mucus on the surface of the seeds was washed with clear water, and the seeds were placed in a constant temperature box at $28^{\circ} \mathrm{C}$ for germination. When the root length of a seed reached $0.5-1.0 \mathrm{~cm}$, it was seeded in a seedling tray with 50 holes and placed in a greenhouse at approximately $30^{\circ} \mathrm{C}$ for $7-10 \mathrm{~d}$ for seedling cultivation ${ }^{[18]}$. The scion seedling raising method was the same as that for the rootstock, with the difference being that it needed to be sown two days earlier than the rootstock.

When the rootstock seedlings had two cotyledons that were entirely flattened and the second true leaf had grown, it indicated that the seedling age was suitable for splice mechanical grafting. The rootstock was cut evenly along the central axis of the development direction of the cotyledon. The area was observed with a Leica M205FA body microscope (Company: Leica Mikrosysteme Vertrieb GmbH, Address: Ernst-Leitz-Strasse 17-37, Wetzlar, 35578 Germany ) to obtain the cavity structure image and geometric feature information of the growth point ${ }^{[16]}$, as shown in Figure 1. $\alpha_{0}$ and $\alpha_{1}$ denote the critical cutting angle and actual cutting angle of the rootstock, respectively.

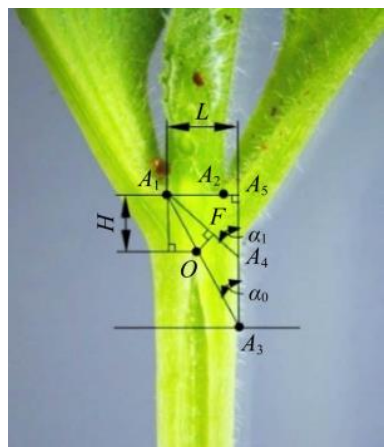

Figure 1 Cross-section of growing point and rootstock cavity

The feature information of the rootstock cavity includes the vertex $O$ of the cavity, left base point $A_{1}$ and right base point $A_{2}$ of the growth point, height $H$ of the stem node, distance $L$ from the left base point to the right edge of the stem, and critical cutting angle $\alpha_{0}$. Among them, the stem node refers to the part from the top of the cavity to the base of the growing point, and the critical cutting angle refers to the angle between the direction from the left base of the growing point $A_{1}$ to the top of the cavity $O$ and the vertical direction. The above feature information was measured and analyzed, the vertex $O$ of the cavity was used as the origin, and a coordinate system was established thereon, so as to obtain the structural feature parameters of the rootstock cavity (as shown in Table 1).

Table 1 Characteristic parameters of rootstock cavity internal structure

\begin{tabular}{|c|c|c|c|c|c|c|c|}
\hline \multirow{2}{*}{ Species } & \multicolumn{2}{|c|}{ Left base point of growth point $A_{1}$} & \multicolumn{2}{|c|}{ Right base point of growth point $A_{2}$} & \multirow{2}{*}{$\begin{array}{l}\text { Height of } \\
\text { stem node } \\
\mathrm{H} / \mathrm{mm}\end{array}$} & \multirow{2}{*}{$\begin{array}{l}\text { Distance from left base point } \\
\text { to the right edge of the stem } \\
\qquad L / \mathrm{mm}\end{array}$} & \multirow{2}{*}{$\begin{array}{l}\text { Critical cutting } \\
\text { angle } \\
\alpha_{0} /\left(^{\circ}\right)\end{array}$} \\
\hline & $X_{1} /$ pixel & $Y_{1} /$ pixel & $X_{2} /$ pixel & $Y_{2} /$ pixel & & & \\
\hline Cucurbita moschata & -97.18 & 273.36 & 106.64 & 271.27 & $1.82 \pm 0.06$ & $2.52 \pm 0.12$ & 19.57 \\
\hline Calabash gourd & -78.08 & 178.85 & 86.23 & 188.85 & $1.58 \pm 0.05$ & $2.23 \pm 0.10$ & 23.58 \\
\hline
\end{tabular}

Note: \pm means standard deviation; The number of test samples is 50 .

It can be seen from Table 1 that the heights $H$ of the stem nodes of cucurbita moschata and calabash gourd are $1.82 \mathrm{~mm}$ and $1.58 \mathrm{~mm}$, respectively, and the $L$ values are $2.52 \mathrm{~mm}$ and $2.23 \mathrm{~mm}$, respectively. This indicates that the range from the vertex of the cavity of the cucurbita moschata to the left and right base points of the growth point is relatively large, which is conducive to the formation of incision cutting. However, owing to the difficulty in cutting calabash gourd, it is easy to cut the cavity. Therefore, it is necessary to strictly control the seedling age of the calabash gourd to increase the height of the stem nodes, to ensure that the calabash gourd seedlings can be safely cut. The critical cutting angles $\alpha_{0}$ for both plants are $19.57^{\circ}$ and $23.58^{\circ}$, respectively. It is necessary to further study the optimal cutting angle for the rootstock and scion matched grafting ${ }^{[23,24]}$.

Based on the results of the above measurements for $L$, the vertex $O$ of the rootstock cavity was selected as the origin, and a plane rectangular coordinate system was established based on $O$. In the established coordinate system, the left base point $A_{1}$ and right base point $A_{2}$ of the growth point of the cucurbita moschata and calabash gourd were respectively constructed, so as to obtain the limit range of the critical cutting angle of the rootstock, as shown in Figure 2.

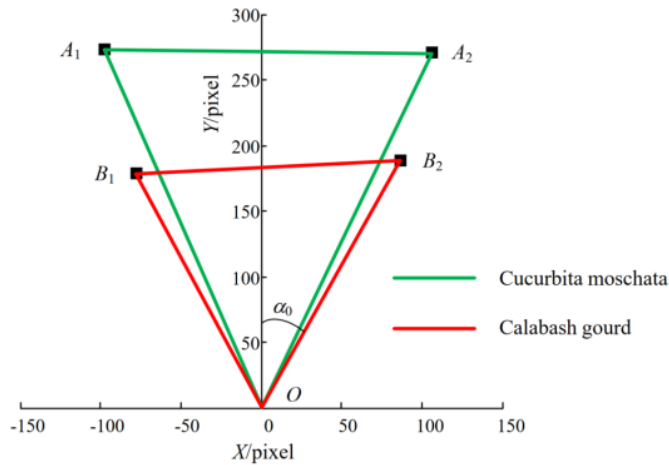

Note: $O$ is the vertex of the pith cavity; $A_{1}$ and $A_{2}$ are the left and right base points of the growth point of the cucurbita moschata, respectively, and $B_{1}$ and $B_{2}$ are the left and right base points of the growth point of the calabash gourd, respectively.

Figure 2 Critical cutting angles of rootstock seedling 
The technical requirements for cutting the rootstock are as follows. First, to avoid damage to the rootstock cotyledon during cutting, it is necessary to complete the cutting operation between the left base point $A_{1}$ and right base point $A_{2}$ of the growth point, i.e., the coordinates $A_{1}$ and $A_{2}$ are the critical points of the cutting operation. Second, to avoid cutting the rootstock cavity, the cutting path must be far away from the vertex $O$ of the cavity, and it must be ensured that the cutting path can pass through the left base point or right base point, to ensure that the growth point is completely cut off. Third, the length of the joint surface of the rootstock and scion should be as long as possible, to increase the contact area for healing and improve the survival rate of the grafted seedlings. Therefore, the effective range of the cutting operation is between the left and right base points of the growth point and the vertex of the cavity. Operations beyond this range will cause damage to the cotyledon or cut the cavity, resulting in the failure of the rootstock cutting.

\subsection{Cutting angle matching of rootstock and scion}

A cut of 10-15 mm of the cotyledon hypocotyls of the scion is required to form a wedge-shaped incision in the splice method, as shown in Figure 3.

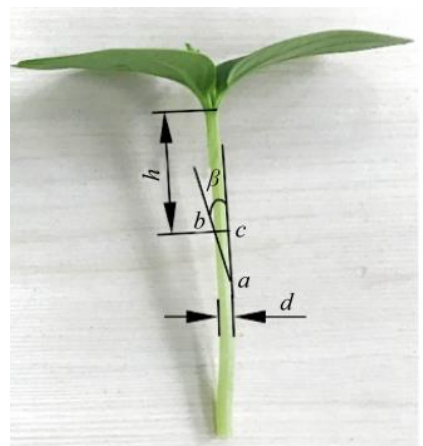

Note: $d$ is the length of the short axis of the scion; $\beta$ is the cutting angle of the scion; $h$ is the cutting height of the scion.

Figure 3 Analysis of cutting angle of scion

The scion cutting angle $\beta$ refers to the angle between the incision of stem and vertical direction and is generally in the range of $20^{\circ}-30^{\circ}$. The smaller the cutting angle, the larger the available cutting surface area; however, if the cutting surface formed after cutting is too thin, it will reduce the pressure bearing capacity of the scion cutting surface, leading to the damage of the scion caused by the grafted seedlings during clamping. If the cutting angles of the rootstock and scion are the same, the cutting length of the scion will be much smaller than that of the rootstock. Therefore, it is necessary to ensure that cutting angles of the rootstock and the scion are completely matched, to provide basic consistency in the section length.

According to Figures 1-3, we can obtain geometry equations for the rootstock and scion sections.

The cut length of rootstock $L_{A 1 A 4}$ can be determined as follows:

$$
L_{A_{1} A_{4}}=\frac{L}{\sin \alpha_{1}}
$$

The distances from the vertex of the cavity to the tangent $L_{O F}$ can be determined as follows:

$$
\begin{gathered}
L_{O F}=L_{O A_{1}} \cdot \sin \left[\left(\frac{\pi}{2}-\alpha_{0}\right)-\left(\frac{\pi}{2}-\alpha_{1}\right)\right] \\
L_{O A_{1}}=\frac{H}{\cos \alpha_{0}}
\end{gathered}
$$

According to Equations (2) and (3), we can obtain an equation as follows:

$$
L_{O F}=H \cdot \frac{\sin \left(\alpha_{1}-\alpha_{0}\right)}{\cos \alpha_{0}}
$$
follows:

The cutting length of the scion $L_{a b}$ can be determined as

$$
L_{a b}=\frac{d}{\sin \beta}
$$

where, $L$ is the distance from the left base point to the right side of the stem, mm; $H$ is the height of the stem node, mm; $\alpha_{0}$ is the critical cutting angle of rootstock, $\left(^{\circ}\right) ; \alpha_{1}$ is the actual cutting angle of the rootstock, $\left({ }^{\circ}\right) ; \beta$ is the cutting angle of the scion, $\left({ }^{\circ}\right)$; and $d$ is the length of the short axis of the scion, $\mathrm{mm}$.

The shape of the scion and rootstock will be more complicated after fitting, increasing the difficulty of calculation, and easily leading to inaccurate results. Therefore, the ratio of the length of the two cutting surfaces is selected to represent the fitting effect of the rootstock and scion and is used to evaluate the cutting quality of the mechanism.

The fitting rate of the rootstock and scion sections is determined as follows:

$$
P=\frac{L_{a b}}{L_{A_{1} A_{4}}} \times 100 \%
$$

According to Equations (1)-(6), a value of $P$ can be obtained as follows:

$$
P=\frac{d \cdot \sin \alpha_{1}}{L \cdot \sin \beta} \times 100 \%
$$

Using the grafting of cucumber and cucurbita moschata as an example, the matching relationship between the cutting angles was analyzed. According to the requirements of grafting, the closer

\begin{tabular}{|c|c|c|c|c|c|c|}
\hline \multirow[b]{2}{*}{$\begin{array}{c}\text { Cutting angle } \\
\alpha_{1} /\left(^{\circ}\right)\end{array}$} & \multicolumn{3}{|c|}{ Cucurbita moschata seedlings } & \multirow{2}{*}{$\begin{array}{c}\text { Cutting angle } \\
\beta /\left(^{\circ}\right)\end{array}$} & \multicolumn{2}{|c|}{ Cucumber seedlings } \\
\hline & $\begin{array}{c}\text { Distance from left base point } \\
\text { to the right side of stem } \\
L / \mathrm{mm}\end{array}$ & $\begin{array}{l}\text { Cutting length } \\
L_{A 1 A 4} / \mathrm{mm}\end{array}$ & $\begin{array}{l}\text { Distance from vertex } \\
\text { to the tangent of cavity } \\
L_{O F} / \mathrm{mm}\end{array}$ & & $\begin{array}{l}\text { Length of short-axis } \\
\qquad d / \mathrm{mm}\end{array}$ & $\begin{array}{l}\text { Cutting length } \\
L_{a b} / \mathrm{mm}\end{array}$ \\
\hline 20 & & $7.37 \pm 0.12$ & $0.06 \pm 0.02$ & 20 & & $5.91 \pm 0.21$ \\
\hline 25 & & $5.96 \pm 0.19$ & $0.27 \pm 0.06$ & 25 & & $4.78 \pm 0.15$ \\
\hline 30 & $2.52 \pm 0.12$ & $5.04 \pm 0.18$ & $0.36 \pm 0.05$ & 30 & $2.02 \pm 0.15$ & $4.04 \pm 0.13$ \\
\hline 35 & & $4.39 \pm 0.15$ & $0.52 \pm 0.04$ & 35 & & $3.52 \pm 0.17$ \\
\hline 40 & & $3.92 \pm 0.16$ & $0.68 \pm 0.05$ & 40 & & $3.14 \pm 0.20$ \\
\hline
\end{tabular}
the cut length, the higher the survival rate of grafting seedlings. The cut length can be controlled by changing the cutting angle. The relevant cutting data for the cucurbita moschata and cucumber seedlings were statistically analyzed at cutting angles of $20^{\circ}, 25^{\circ}$, $30^{\circ}, 35^{\circ}$, and $40^{\circ}$, and the results are shown in Table 2.

Table 2 Cutting angle matching analysis of rootstock and scion

Note: There were 50 test samples in each group. 
As shown in Table 2, as the cutting angle of the cucurbita moschata increased, the length of the cutting surface gradually decreased, and the effective distance from the vertex of the cavity to the cutting surface increased. When the cutting angle was $20^{\circ}$, the $L_{O F}$ was only $0.06 \mathrm{~mm}$, and there was a risk that the cavity could be cut; thus, $20^{\circ}$ was not a suitable cutting angle for the cutting operation. When the cutting angle was greater than $35^{\circ}$, the $L_{O F}$ was greater than $0.5 \mathrm{~mm}$, and the section length was approximately $4 \mathrm{~mm}$, i.e., not conducive to the survival of grafted seedlings. When the cutting angle was in the range of $25^{\circ}-30^{\circ}$, the section length was in the range of 5.04-5.96 mm, and $L_{O F}$ was in the range of $0.27-0.36 \mathrm{~mm}$, meeting the requirements for the cutting operation. When the cutting angle of the cucumber was $20^{\circ}$, its section length was $5.91 \mathrm{~mm}$. When the cutting angle was $25^{\circ}-40^{\circ}$, the section length was less than $5 \mathrm{~mm}$. The comprehensive analysis showed that when the cutting angles of the cucurbita moschata and cucumber seedlings were $25^{\circ}$ and $20^{\circ}$, respectively, the fitting rate of the cutting surfaces of the rootstock and scion could reach $99.04 \%$, meeting the grafting requirements.

\subsection{Determination of cutting method}

It is challenging to control the angle of the leaf and petiole of the rootstock. Thus, it is easy to cut them during the operation. Therefore, it is necessary to allow the cotyledon pressing mechanism to keep the cotyledon down to avoid the cutting trajectory of the cutter and improve the accuracy and stability of the cutting operation, as shown in Figure 4.

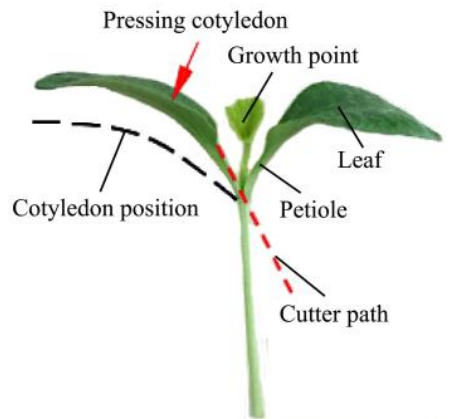

Figure 4 Analysis of cutting work of rootstock seedling

There is a variety of cutting methods for rootstock, including linear cutting and rotary cutting. Rotary cutting can obtain a higher cutting speed and better cutting quality. In contrast, linear cutting requires a long output distance to achieve a higher cutting speed; thus, the designed structure for the cutting mechanism must be relatively large. Therefore, rotary cutting is selected as the method for rootstock cutting.

\section{Design of the cutting mechanism}

\subsection{Structure and working principle}

It can be seen from the analysis that if the direction of rotary cutting is perpendicular to the direction of the growth of the cotyledon, it will increase the difficulty of separating the cotyledons from the growth point, and is not conducive to cutting. If the cutting is conducted from the bottom to the top along the growth direction of the cotyledon, the cutting path will be formed from the petiole of the cotyledon to the left base of the growth point, allowing the cotyledon and the growth point to be cut more entirely, and forming a high-quality section.

As shown in Figure 5, the rootstock cutting mechanism includes a bracket, tool apron, fixed plate, $X Y$-direction adjusting platform, pressing cotyledon cylinder, pressing cotyledon plate, rotating cylinder, cutter, and handle ${ }^{[16]}$.
The spatial position of the cutter rotation center can be accurately adjusted according to the cutting model of the working object. By using the $X Y$ direction adjustment platform to adjust the position of the cutter rotation center in the height (vertical) and horizontal directions, the cutter trajectory can meet the cutting model for the rootstock seedlings. The adjustment range of the $X Y$ platform in the height and horizontal directions is $\pm 6.5 \mathrm{~mm}$, and the adjustment accuracy is $0.01 \mathrm{~mm}$. The seeding pressing and growth point cutting of the cotyledon are driven by the seeding pressing cylinder and rotating cylinder. The seeding pressing cylinder extends to drive the seeding pressing. The remaining cotyledon is pressed down to completely separate the cotyledon and growth point. The rotary cylinder drives the tool handle and cutter to rotate $180^{\circ}$ from bottom to top, to complete the removal of the cotyledon and growth point.

Considering that for different varieties of rootstock seedlings, the internal medullary cavity structures are different; as such, the cutting model should also differ. When the variety of the rootstock is changed, the cutter rotation center of the cutting mechanism is adjusted accurately according to the cutting model of the working object, ensuring the cutting success rate. Therefore, it is necessary to adjust the position of the cutter rotation center according to the cutting models for different types of rootstock, so as not to affect the cutting success rate.

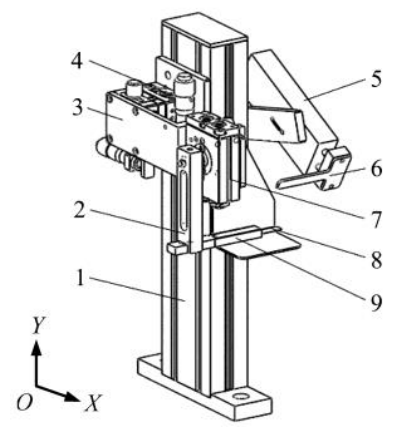

1. Bracket 2. Handle 3. Fixed plate 4. $X Y$ direction-adjusting platform 5. Pressing a cotyledon cylinder 6. Pressing the cotyledon plate 7. Rotating cylinder 8. Cutter 9. Tool apron.

Figure 5 Structure diagram of rootstock cutting mechanism

Working process: First, the spatial position of the cutting tool operation is adjusted. When the seedlings are transported to the cutting station by picking and transporting mechanism, the pressing cotyledon cylinder extends to drive the pressing cotyledon plate to press the reserved cotyledon down and keep it away from the cutting track, so as to avoid cutting injury. The rotating cylinder drives the handle and cutter to complete a $180^{\circ}$ cutting action from bottom to top. The cutting operation track successfully avoids the previously reserved cotyledon and completely cuts another cotyledon and growth point to form a cutting surface, thereby completing a cutting process. The pressing cotyledon cylinder and rotating cylinder are installed on both sides of the bracket, respectively. By using the $X Y$ adjusting platform, the position of the cutter in the vertical plane can be precisely adjusted, and different cutting angles can be completed. The tool apron is connected and fixed with the output shaft of the rotary cylinder through the tool handle, and the cutter is fixed in the cutter base through strong magnetic absorption.

\subsection{Geometric model of cutting}

When rootstock is cut, the pressing cotyledon plate presses the cotyledon, providing a safe working space for the cutter and ensuring the safety and stability of the cotyledon cutting. As a result of the arc-shaped section forms after rotary cutting, the closer 
the section is to the plane, the easier it is to fit the rootstock and scion. Therefore, it is necessary to determine the relative positional relationships between the pressing cotyledon plate, center of the rotary cutting, and center of the section, as shown in Figure 6.

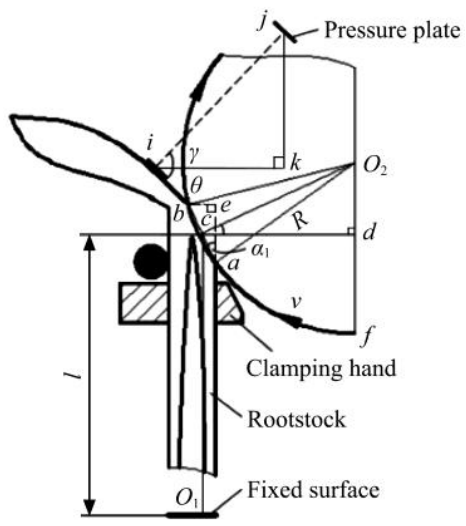

Note: $l$ is the height of the center section, $\mathrm{mm} ; R$ is the cutting radius, $\mathrm{mm} ; \alpha_{1}$ is the cutting angle, $\left({ }^{\circ}\right) ; \gamma$ is the angle of the pressing cotyledon, $\left({ }^{\circ}\right)$; and $\theta$ is the angle between the petiole pressing cotyledon and the horizontal direction, $\left({ }^{\circ}\right)$.

Figure 6 Geometric model of cutting rootstock

Taking the intersection $O_{1}$ of the center of the section and installation surface of the equipment as the origin, a plane rectangular coordinate system is established based on $O_{1}$, and its cutting geometry model is shown as follows.

The coordinates of the cutter rotation center $\mathrm{O}_{2}$ are as follows:

$$
\left\{\begin{array}{l}
x_{O_{1}}=R \cos \alpha_{1} \cdot \sqrt{1-\frac{L^{2}}{4 R^{2} \cdot \sin ^{2} \alpha_{1}}} \\
y_{O_{1}}=R \sin \alpha_{1} \cdot \sqrt{1-\frac{L^{2}}{4 R^{2} \cdot \sin ^{2} \alpha_{1}}}+l
\end{array}\right.
$$

The coordinates of center $j$ of the pressing cotyledon plate are as follows:

$$
\left\{\begin{array}{l}
x_{j}=l_{i j} \cos \gamma-l_{i b} \cdot \cos \theta-\frac{L}{2} \\
y_{j}=l_{i j} \sin \gamma+l_{i b} \cdot \sin \theta+\frac{L}{\tan \alpha_{1}}+l
\end{array}\right.
$$

The dent depth of the section $\Delta$ is determined as follows:

$$
\Delta=R-\sqrt{R^{2}-\frac{L^{2}}{4 \sin ^{2} \alpha_{1}}}
$$

where, $R$ is the cutting radius, $\mathrm{mm} ; l_{i j}$ is the pressing distance, $\mathrm{mm}$; $l_{\mathrm{ib}}$ is the distance from the pressing point to the left base point, mm; $L$ is the distance from the left base point of the growing point to the right side of the stem, mm; and $\alpha_{1}$ is the cutting angle of the rootstock, $\left({ }^{\circ}\right)$.

According to the corresponding requirements for pressing the cotyledon and the cutting operation, the design constant $\gamma$ is $45^{\circ}, \theta$ is $30^{\circ}, h$ is $185 \mathrm{~mm}, l_{i j}$ is $50 \mathrm{~mm}$, and $l_{i b}$ is $10 \mathrm{~mm}$. There exists a certain relationship between the center position of the rotary cutting, cutting radius, and cutting angle. The center position of the pressing seeding plate is related to the cutting angle. The larger the cutting radius is, the smaller the cutting depth is. Taking the cucurbita moschata seedlings as an example, when $L$ is $2.52 \mathrm{~mm}, \alpha$ is $25^{\circ}$ and $R$ is $68 \mathrm{~mm}$, by substituting Equation (4) and Equations (7)-(9), it can be concluded that the dent depth of the section is $0.06 \mathrm{~mm}$, and the distance from the vertex of the cavity to the section is $0.24 \mathrm{~mm}$, meeting the grafting requirements. The cutter rotation center coordinates are $(61.55,215.03)$, and the pressing cotyledon plate center coordinates are $(25.43,228.81)$. Thus, this approach can provide a reference for the precise adjustment of the positions of the cutter and pressing cotyledon plate.

\subsection{Optimization of cutting mechanism parameters}

As shown in Figure 7, the factors influencing the cutting angle of the cutting mechanism include the cutting radius $R$, cutter inclination angle $\omega$, and turning center position $O_{2}$. The cutting radius $R$ represents the distance between the center axis of the rotating cylinder and the center of the cutter. The cutter installation angle $\omega$ is the included angle between the plane where the cutter is located and the plane perpendicular to the handle. The initial point is $0^{\circ}$, the clockwise direction is positive, and the counterclockwise direction is negative. The single-factor influences of the cutting radius and cutter inclination angle on the cutting angle were analyzed ${ }^{[25-27]}$.

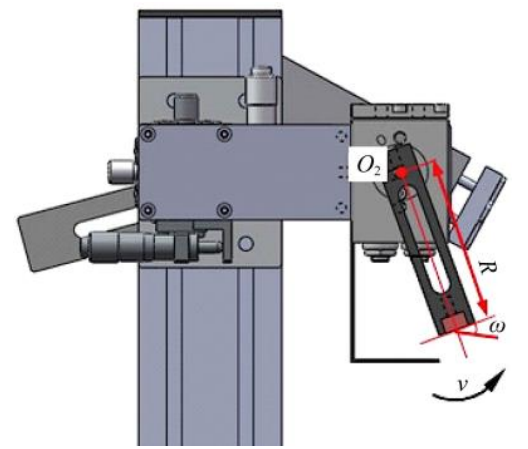

Note: $R$ is the cutting radius; $\omega$ is the cutter inclination angle, and point $\mathrm{O}_{2}$ is the turning center position.

Figure 7 Diagram of cutting parameters

Taking cucurbita moschata as an example, a change rule of the cutting angle in the range of $66-76 \mathrm{~mm}$ was analyzed. The specific results are shown in Figure 8a. The cutting angle increases with the increase of the cutting radius, as consistent with the theoretical analysis. The coefficient of determination $R^{2}$ of the regression equation is 0.9844 , indicating that the cutting radius has a significant effect on the cutting angle. When the cutting radius are $66 \mathrm{~mm}$ and $72 \mathrm{~mm}$, the corresponding cutting angles are $20.64^{\circ}$ and $31.17^{\circ}$, respectively, indicating that when the cutting radius is less than $66 \mathrm{~mm}$ or more than $72 \mathrm{~mm}$, the cutting angle does not meet the grafting requirements. When the cutting angle is $25^{\circ}$, the cutting radius is $68.25 \mathrm{~mm}$, i.e., basically consistent with the cutting radius in Section 3.2. The change rule of the cutting angle in the range of $-15^{\circ}-15^{\circ}$ was analyzed, and the results were shown in Figure $8 \mathrm{~b}$. The cutting angle decreases with an increase in the cutter inclinations, and the coefficient of determination $R^{2}$ of the fitting regression equation is 0.9279 , indicating that the cutter inclinations have a significant effect on the cutting angle. When the cutter inclinations are $0^{\circ}$ and $15^{\circ}$, the corresponding cutting angles are $30^{\circ}$ and $25.6^{\circ}$, respectively. When the cutting angle is greater than $15^{\circ}$ or less than $0^{\circ}$, the result will not be within the range of cutting requirements; thus, the cutter inclinations is set between $0^{\circ}-15^{\circ}$.

In the cutting process, it was found that setting the cutting angle by adjusting the cutting radius and inclinations of the cutter was a very complicated process. After changing any factor, the cutting path changes; therefore, it cannot be guaranteed that the cutting path can pass the left or right base point of the growth point at any time. This results in the absence of a complete cutting of the growth point and affects the cutting quality. Therefore, the left or right base point of the growth point must be taken as the reference point. Only by ensuring that this point is reached can it be ensured that the growth point is completely cut off. Therefore, 
by setting the cutting radius $(R=68.25 \mathrm{~mm})$ and cutter inclination $\left(\omega=0^{\circ}\right)$ as fixed values and adjusting the spatial position of the cutter rotation center by using the $X Y$ adjustment platform, the rootstock cutting angle can be changed so that the cutting path can pass the left or right base point of the growth point at any time, thereby simplifying the rootstock cutting geometry model.

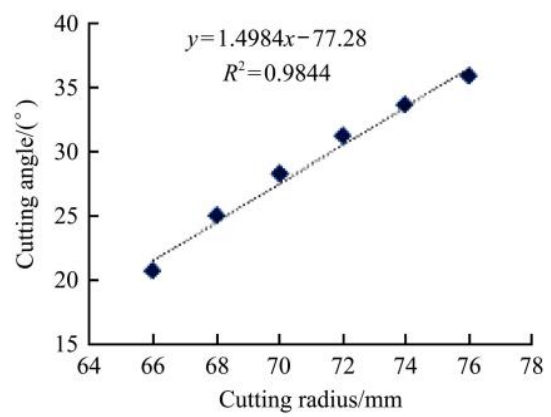

a. The influence of cutting radius on cutting angle

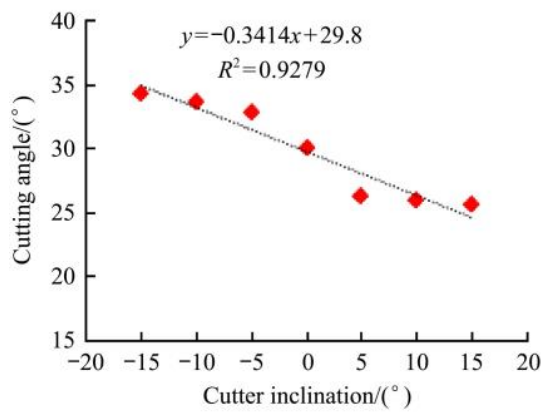

b. The influence of cutter inclination on cutting angle

Figure 8 Single-factor test results of cutting angle

\section{Experiment and analysis}

\subsection{Experiment purpose}

Through the above analysis, it is known that different lengths of the section can be obtained by changing the location of the center space. To verify the design rationality and cutting performance of the rootstock cutting mechanism, the success rate of pressing the cotyledon seedlings, cutting success rate, and accuracy rate were taken as evaluation indexes, and the research and analysis were conducted in the context of the rootstock cutting test.

The success rate of pressing a cotyledon $M$ is as follows:

$$
M=\frac{N}{F} \times 100 \%
$$

The cutting success rate $Q$ is as follows:

$$
Q=\frac{E}{F} \times 100 \%
$$

The cutting accuracy $T$ is as follows:

$$
T=\left(1-\frac{\left|\alpha_{2}-\alpha_{1}\right|}{\alpha_{2}}\right) \times 100 \%
$$

where, $N$ represents the successful number of pressed cotyledons; $E$ is the number of successful cuttings at the growth point; $F$ is the total number of rootstock cutting tests; $\alpha_{2}$ is the preset cutting angle, $\left({ }^{\circ}\right) ; \alpha_{1}$ is the actual cutting angle, $\left({ }^{\circ}\right)$.

\subsection{Experimental method}

Age-appropriate cucurbita moschata seedlings were selected as the research object and were used in the designed rootstock cutting mechanism for the test, as shown in Figure 9.

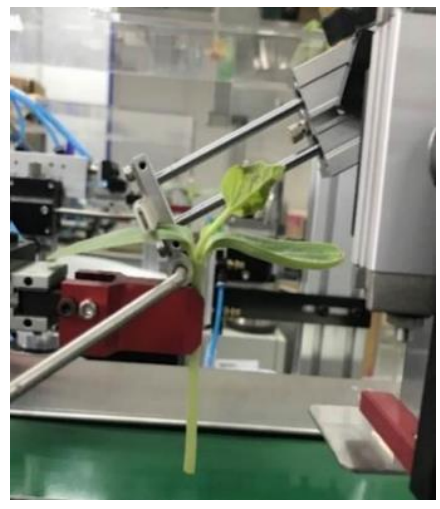

a. Pressing cotyledon

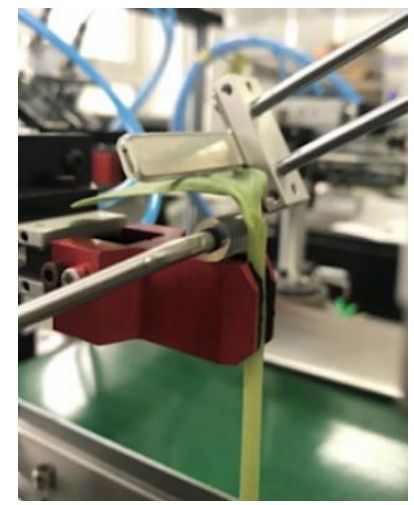

b. Cutting growing point

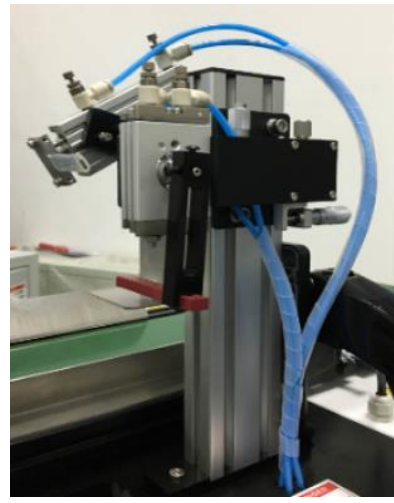

c. Cutting mechanism

Figure 9 Cutting test of rootstocks

In the experiment, the relative positions of the rootstock seedling picking and transporting mechanism and cutting mechanism were initially determined. The preset cutting angle was set to $25^{\circ}$, and the position of the cutter rotation center was adjusted using the $X Y$ adjusting platform. Then, by using the pressure-regulating valve, the air inlet pressures of the pressing cotyledon cylinder and rotating cylinder were set to $0.4 \mathrm{MPa}$, and the cutting radius was set as $68.25 \mathrm{~mm}$. The test was divided into three groups, with 50 seedlings in each group.

The experimental method was as follows. The rootstock seedling was placed into a feeding seedling positioning mechanism, and the seedling picking and transporting mechanism was used to clamp and transport the rootstock to the cutting station. The seedling pressing cylinder extended out the air pressure seedling plate to keep the cotyledon pressed, and to analyze the success rate and reason(s) for seedling pressing. After the cotyledon treatment, the air cylinder was rotated to ventilate, and the cutter was driven to cut at the cotyledon and growth point. Then, the rootstock seedling was removed, the cutting length and $\mathrm{L}$ value were measured using a Vernier caliper, and the cutting success rate and cutting accuracy were analyzed. The section fitting rate of the rootstock and cotyledon is an important index reflecting the performance of the cutting mechanism. According to the length of the scion cut, as measured in Section 2.2, the fitting rate of the cutting surfaces of the rootstock and scion was comprehensively calculated.

\subsection{Results and analysis}

The cutting test results for the rootstock are shown in Table 3. The success rate of pressing the cotyledons was $96.67 \%$. The reason for the failure was that the rootstock cotyledon and growing point were too large. The cotyledon and the growing point were pressed down simultaneously during the pressing operation, but the cutting operation was not affected. By adjusting the installation angle and position of the pressing cotyledon cylinder, the success 
rate of the cotyledon seedling pressing was improved in a follow-up test. The success rate of cutting was $98 \%$. The reason for the failure was that the feeding seedling direction and overall feeding seedling height of the cotyledons were inaccurate, leading to incomplete or excessive cutting of the growth point. The cutting accuracy was $96.8 \%$ and the cutting fit rate of the rootstock and scion was $98.61 \%$, meeting the requirements for mechanical grafting. It is feasible to change the cutting angle by adjusting the position of the cutting center. This method can accurately draw the left base point of the growth point into the cutting path, thus verifying the rationality of the cutting mechanism design and structural parameters.

Table 3 Cutting test results of rootstock

\begin{tabular}{|c|c|c|c|c|c|c|c|}
\hline Group & $\begin{array}{l}\text { Rootstock } L \text { value } \\
/ \mathrm{mm}\end{array}$ & $\begin{array}{l}\text { Cutting length } \\
\text { of rootstock } \\
L_{A 1 A 4} / \mathrm{mm}\end{array}$ & $\begin{array}{c}\text { Actual cutting } \\
\text { angle } \\
\alpha_{1} /\left(^{\circ}\right)\end{array}$ & $\begin{array}{c}\text { The success rate of } \\
\text { seedling pressing } \\
M / \%\end{array}$ & $\begin{array}{c}\text { Cutting success } \\
\text { rate } \\
Q / \%\end{array}$ & $\begin{array}{c}\text { Cutting } \\
\text { accuracy } \\
T / \%\end{array}$ & $\begin{array}{c}\text { Fitting rate of cutting } \\
\text { surface of rootstock and } \\
\text { scion } P / \%\end{array}$ \\
\hline 1 & $2.44 \pm 0.13$ & $6.03 \pm 0.12$ & $23.83 \pm 0.15$ & 94 & 96 & 95.32 & 98 \\
\hline 2 & $2.46 \pm 0.15$ & $5.99 \pm 0.17$ & $24.21 \pm 0.13$ & 96 & 98 & 96.84 & 98.66 \\
\hline 3 & $2.49 \pm 0.11$ & $5.96 \pm 0.16$ & $24.56 \pm 0.12$ & 100 & 100 & 98.24 & 99.16 \\
\hline Mean & 2.46 & 5.99 & 24.20 & 96.67 & 98 & 96.8 & 98.61 \\
\hline
\end{tabular}

Note: there are 50 test samples in each group.

\section{Conclusions}

To solve problems such as the incomplete removal of rootstock growth points and the low precision of cutting mechanisms in existing grafting machines, an internal cavity structure model of cucurbita moschata and calabash gourd seedings was established using image processing technology, and their critical cutting angles were determined as $19.57^{\circ}$ and $23.58^{\circ}$, respectively. The cutting angles of the matching grafting for the cucurbita moschata and cucumber seedlings were $25^{\circ}$ and $20^{\circ}$, respectively, and the fitting rate of the cutting surface of rootstock and scion was $99.04 \%$. Rotary cutting was determined as the operation mode. A rootstock cutting mechanism was designed with combining pressing and rotating cutting, and a geometry model was constructed for rotating the cutting for the rootstock cotyledons and growing points. The positions of the rotating cutting center and pressing plate were determined, and a geometry equation was obtained for the cutting dent depth. In addition, through single-factor influence tests of the cutting radius and cutter inclination angle, a method of adjusting the cutting center to change the cutting angle was determined. This method is more conducive to improving the cutting accuracy for rootstock and simplify the cutting geometry model for rootstock. According to the performance test of the rootstock cutting mechanism, the success rate of pressing the cotyledons is $96.67 \%$, and the success rate of cutting is $98 \%$. The main reason for the failure in pressing the cotyledons is that the cotyledons and growth points are too large, but these factors do not affect the quality of the cutting operation. The main reason for the cutting failure is that the feeding seedling direction of the cotyledons and height of the entire feeding seedlings are inaccurate, leading to incomplete or excessive cutting. The cutting accuracy is $96.8 \%$ and the fitting rate of the cutting surface of the rootstock and scion is $98.61 \%$, meeting the requirements of mechanical grafting. The results of this study can provide a reference for the design of a cutting mechanism for a grafting robot.

\section{Acknowledgements}

This work was funded by the Beijing Academy of Agriculture and Forestry Sciences Innovation Ability Project (Grant No. KJCX20180422), the Key Research and Development projects in Ningxia Hui Autonomous Region (Grant No. 2018BBF02024), and the National Key Technology Research and Development Program of China (Grant, No. 2013AA102406). The authors wish to thank their generous financial assistance.

\section{[References]}

[1] Pardo-Alonso J L, Carreño-Ortega Á, Martínez-Gaitán C C, Golasi I, Gómez-Galán M. Conventional industrial robotics applied to the process of tomato grafting using the splicing technique. Agronomy, 2019; 9(12): 880. doi: 10.3390/agronomy9120880.

[2] Zhu C Y, Yue D J. Production status and technology trend of vegetable seedling industry in China. Agricultural Engineering Technology, 2019; 39(13): 34-38. (in Chinese)

[3] Liu M C, Ji Y H, Wu Z H, He W M. Current situation and development trend of vegetable seedling industry in China. China Vegetables, 2018; 11: 1-7. (in Chinese)

[4] Huang Y, Kong Q S, Chen F, Bie Z L. The history, current status and future prospects of vegetable grafting in China. Acta Horticulturae, 2015; 1086: 31-39.

[5] Yang Y M, Wang G B. Research on the aging of rural population in China. Shanxi Agricultural Economy, 2018; 2: 1-3. (in Chinese)

[6] Jiang K, Zheng W G, Zhang Q, Guo R, Feng Q C. Development and experiment of vegetable grafting robot. Transactions of the CSAE, 2012; 28(4): 8-14. (in Chinese)

[7] Chen X, Gong Y, Zhang X, Liu D J, Wang, G. Development status of machinery and equipment for watermelon and melon production. China Cucurbits and Vegetables, 2019; 32(8): 65-68. (in Chinese)

[8] Kobayashi K, Sasaya S. Study on automation of seedlings feeding for grafting robot for cucurbitaceous vegetables (Part 2). Agricultural Machinery and Food Engineers, 2007; 69(5): 70-77.

[9] Chiu Y C, Chen S, Chang Y C. Development of a circular grafting robotic system for watermelon seedlings. Appl. Eng. Agric, 2011; 10: 95-102.

[10] Kim H M, Hwang S J. Comparison of pepper grafting efficiency by grafting robot. Protected Horticulture and Plant Factory, 2015; 24(2): $57-62$.

[11] Kang D H, Lee S Y, Kim J K, Park M J, Son J K, Yun S W Development of an automatic grafting robot for fruit vegetables using image recognition. Protected Horticulture and Plant Factory, 2019; 28(4): 322-327.

[12] Ohkoshi T, Kobayashi K. Development of automatic seedling feeding device for cucurbits grafting robot (Part 1)-Evaluation of automatic stock feeder. Journal of the Japanese Society of Agricultural Machinery and Food Engineers, 2013; 75(2): 100-107.

[13] Zhang P, Zhang L N, Liu D, Wu H X, Jiao B. Research status of agricultural robot technology. Agricultural Engineering, 2019; 9(10): $1-12$.

[14] Li D D, Shi Y, Li H B, Han W, Duan Y L, Wu W B. Review on the progress of agricultural robot research. China Agricultural Informatics, 2018; 30(6): 1-17. (in Chinese)

[15] Xie Z J, Gu S, Chu Q, Li B, Fan K J, Yang Y L, et al. Development of a high-productivity grafting robot for Solanaceae. Int J Agric \& Biol Eng, 2020; 13(1): 82-90.

[16] Zhu C X, Jiang W, Liu W, Wang W, Du J W, Hao L F, et al. Research progress in cucumber grafting seedling raising technology. Journal of Northern Agriculture, 2019; 47(2): 115-118. (in Chinese)

[17] Jiang K. Study on mechanism and experimental device of splice 
mechanical grafting of cucurbit. $\mathrm{PhD}$ dissertation. Harbin: Northeast Agricultural University, 2019; 132p. (in Chinese)

[18] Mu Y H, Gu S, Ma Z Y. Experimental analysis on biomechanical properties of cucurbits. Transactions of the CSAE, 2012; 28(4): 15-20. (in Chinese)

[19] Hassell R L, Memmott F, Liere D G. Grafting Methods for Watermelon Production. Hortscience, 2008; 43(6): 1677-1679.

[20] Zhang L, He H, Wu C Y. Vision method for measuring grafted seedling properties of vegetable grafted robot. Transactions of the CSAE, 2015; 31(9): 32-38. (in Chinese)

[21] Zhang J, Zhao H, Jia J, Zhang C H, Yu C L, Yu Q C. Vision driven prototype system of automatic grafting machine. Journal of Zhejiang Agricultural Sciences, 2018; 59(10): 1763-1766. (in Chinese)

[22] Wang Z L, Cheng X J, You W S. Design of automatic seedling control system for vegetable grafting machine based on machine vision. Journal of Anhui Agricultural Sciences, 2019; 47(7): 218-220. (in Chinese)
[23] He L Y, Cai L Y, Wu C Y. Vision-based parameters extraction of seedlings for grafting robot. Transactions of the CSAE, 2013; 29(24): 190-195. (in Chinese)

[24] Cui Y J, Wang X X, Xu L Q, Chen T, Li S H, Fu L S. Automatic detection for external features of grafting seedlings based on machine vision. Transactions of the CSAM, 2014; 45(4): 89-95. (in Chinese)

[25] Pardo-Alonso J L, Carreño-Ortega Á, Martínez-Gaitán C C, Callejón-Ferre Á J. Combined influence of cutting angle and diameter differences between seedlings on the grafting success of tomato using the splicing technique. Agronomy, 2019; 9(1): 5. doi: 10.3390/agronomy9010005.

[26] Tian S B, Song C C, Dong S, Wang R L. Parameter optimization and experiment for cutting device of muskmelon grafting machine. Transactions of the CSAE, 2016; 32(22): 86-92. (in Chinese)

[27] Jiang K, Zheng W G, Zhang Q, Guo R, Feng Q C. Design and experiment of grafting robot cutting device. Journal of Agricultural Mechanization Research, 2012; 34(2): 76-79, 83. 